\title{
Characterisation of iunH gene knockout strain from Mycobacterium tuberculosis
}

\author{
Anne Drumond Villela', Valnês da Silva Rodrigues-Junior ${ }^{1,2,3}$, Antônio Frederico Michel Pinto', \\ Priscila Lamb Wink ${ }^{1,4}$, Zilpa Adriana Sánchez-Quitian ${ }^{1,4}$, Guilherme Oliveira Petersen', \\ Maria Martha Campos ${ }^{2,3,4}$, Luiz Augusto Basso ${ }^{1,2,4}$, Diógenes Santiago Santos ${ }^{1,4} /+$
}

1Pontifícia Universidade Católica do Rio Grande do Sul, Centro de Pesquisas em Biologia Molecular e Funcional, Instituto Nacional de Ciência e Tecnologia em Tuberculose, Porto Alegre, RS, Brasil

${ }^{2}$ Pontifícia Universidade Católica do Rio Grande do Sul,

Programa de Pós-Graduação em Medicina e Ciências da Saúde, Porto Alegre, RS, Brasil

${ }^{3}$ Pontifícia Universidade Católica do Rio Grande do Sul, Instituto de Toxicologia e Farmacologia, Porto Alegre, RS, Brasil

${ }_{4}^{4}$ Pontifícia Universidade Católica do Rio Grande do Sul, Programa de Pós-Graduação em Biologia Celular e Molecular, Porto Alegre, RS, Brasil

BACKGROUND Tuberculosis (TB) is an infectious disease caused mainly by the bacillus Mycobacterium tuberculosis. The better understanding of important metabolic pathways from $M$. tuberculosis can contribute to the development of novel therapeutic and prophylactic strategies to combat TB. Nucleoside hydrolase (MtIAGU-NH), encoded by iunH gene (Rv3393), is an enzyme from purine salvage pathway in M. tuberculosis. MtIAGU-NH accepts inosine, adenosine, guanosine, and uridine as substrates, which may point to a pivotal metabolic role.

OBJECTIVES Our aim was to construct a $M$. tuberculosis knockout strain for iunH gene, to evaluate in vitro growth and the effect of iun $H$ deletion in $M$. tuberculosis in non-activated and activated macrophages models of infection.

METHODS A $M$. tuberculosis knockout strain for iunH gene was obtained by allelic replacement, using pPR27xylE plasmid. The complemented strain was constructed by the transformation of the knockout strain with pNIP40::iunH. MtIAGU-NH expression was analysed by Western blot and LC-MS/MS. In vitro growth was evaluated in Sauton's medium. Bacterial load of non-activated and interferon- $\gamma$ activated RAW 264.7 cells infected with knockout strain was compared with wild-type and complemented strains.

FINDINGS Western blot and LC-MS/MS validated iunH deletion at protein level. The iunH knockout led to a delay in $M$. tuberculosis growth kinetics in Sauton's medium during log phase, but did not affect bases and nucleosides pool in vitro. No significant difference in bacterial load of knockout strain was observed when compared with both wild-type and complemented strains after infection of non-activated and interferon- $\gamma$ activated RAW 264.7 cells.

MAIN CONCLUSION The disruption of $i u n H$ gene does not influence $M$. tuberculosis growth in both non-activated and activated RAW 264.7 cells, which show that $i u n H$ gene is not important for macrophage invasion and virulence. Our results indicated that MtIAGU-NH is not a target for drug development.

Key words: iunH gene - nucleoside hydrolase - gene knockout - Mycobacterium tuberculosis

Tuberculosis (TB) is an infectious disease caused mainly by the bacillus Mycobacterium tuberculosis, and remains one of the world's deadliest contagious diseases. TB incidence is slowly declining each year, however, novel drugs and vaccines are urgently needed to stop global transmission and to prevent the development of drug-resistant strains (WHO 2016). The better understanding of important metabolic pathways from $M$. tuberculosis can contribute to the development of novel therapeutic and prophylactic strategies to combat TB.

doi: 10.1590/0074-02760160462

Financial support: CNPq (304051/1975-06, 520182/99-5, 163507/2014-

7, 304156/2014-0), BNDES (14.2.0914.1), FAPERGS-CAPES (DOCFIX,

05/2013), Decit/SCTIE/MS-MCT-CNPq-FNDCT-CAPES to INCT-TB, CNPq

(441720/2014-5), FAPERGS-CNPq-PRONEX-2009.

+Corresponding author: diogenes@pucrs.br

Received 18 October 2016

Accepted 2 December 2016
Purine nucleotides in $M$. tuberculosis may be formed de novo from simple precursors, or may be obtained by the salvage pathway from preformed purine bases and nucleosides. While the de novo pathway is a high energy demanding process, which involves up to 11 enzymatic steps, the salvage pathway might be the main source to maintain the nucleotide pool under conditions of low energy availability or rapid multiplication (Ducati et al. 2011). Nucleoside hydrolase (MtIAGU-NH), encoded by iunH gene (Rv3393, Gene ID: 887625), is an important enzyme from the purine salvage pathway in $M$. tuberculosis, which catalyses the irreversible hydrolysis of $\mathrm{N}$ glycosidic bond of ribonucleosides forming $\alpha$-D-ribose and the corresponding base (Wink et al. 2013). MtIAGU$\mathrm{NH}$ was shown to have broad substrate specificity, accepting inosine, adenosine, guanosine, and uridine as substrates, which may point to a pivotal metabolic role in M. tuberculosis (Wink et al. 2013). Here, we describe the construction of a $M$. tuberculosis knockout (KO) strain for $i u n H$ gene, in vitro growth studies, and the ef- 
fect of iunH deletion in M. tuberculosis in non-activated and activated macrophage model of infection, comparing with M. tuberculosis H37Rv wild-type (WT) and complemented (CP) strains.

\section{MATERIALS AND METHODS}

Plasmid construction for generation of the knockout strain - A fragment of $1782 \mathrm{bp}$ containing the iunH gene (927 bp) with its flanking region (Fig. 1A) was amplified by polymerase chain reaction (PCR) from $M$. $t u$ berculosis $\mathrm{H} 37 \mathrm{Rv}$ genomic DNA, using primers forward (5'-tttttctagagcagcaggcgatgcgecagg-3') and reverse (5'tttttctagagaccegtcgecggeggtgc-3'), both containing XbaI restriction sites (underlined). The $1782 \mathrm{bp}$ fragment was subsequently cloned into pUC19 using the $\mathrm{XbaI}$ restriction site. The $i u n H$ gene was disrupted by the insertion of a kanamycin cassette from pUC4K into unique internal enzyme restriction site $X c m I$ (New England Biolabs, USA) (Fig. 1B). Insert was released from pUC19 derivative vector by digestion with $\mathrm{XbaI}$ (New England Biolabs, USA), and subcloned into $X b a I$ linearised pPR 27xylE vector (pPR27xylE::iunH kan) (Fig. 1B) (Pelicic et al. 1997).

Plasmid construction for generation of the complemented strain - The iunH gene flanked by about 200 bp upstream and $100 \mathrm{bp}$ downstream, was amplified by PCR from M. tuberculosis H37Rv genomic DNA using primers forward (5'-ttttctagacagcgegagatcgatcttg-3') and reverse (5'-tttttctagacggtggtatctggagggaa-3'), both containing $\mathrm{XbaI}$ restriction sites (underlined), and was cloned into XbaI linearised pNIP40/b (pNIP40::iunH), a mycobacteriophage Ms6-derived integrative vector (Freitas-Vieira et al. 1998).

Construction of the M. tuberculosis knockout strain - Electrocompetent cells were prepared as described (Parish \& Stocker 1998) with some modifications. M. tuberculosis H37Rv strain was grown in $50 \mathrm{~mL}$ of Middlebrook 7H9 (Becton Dickinson, BD, USA) 10\% OADC (oleic acid, albumin, dextrose, and catalase) (BD, USA) $0.05 \%$ tween-80 (Sigma-Aldrich, USA) (liquid medium) to an $\mathrm{OD}_{600}$ of 0.6. Cells were washed two times in $0.05 \%$ tween- 80 , one time in $10 \%$ glycerol containing $0.05 \%$ tween-80, and were suspended in $500 \mu \mathrm{L}$ of $10 \%$ glycerol containing $0.05 \%$ tween- 80 . Aliquots $(200 \mu \mathrm{L})$ of fresh prepared competent cells were electroporated with approximately $2 \mu \mathrm{g}$ of pPR $27 x y l E:: i u n H$ kan plasmid in $0.2 \mathrm{~cm}$ cuvettes with a single pulse $(2.5 \mathrm{kV} ; 25 \mathrm{mF} ; 1000$ ohms). The pPR27xylE plasmid contains a thermosensitive origin of replication, the $x y l E$ reporter gene, and the $s a c B$ counterselectable marker. Bacteria were plated on Middlebrook 7H10 (BD, USA) 10\% OADC (solid medium) containing $25 \mu \mathrm{g} / \mathrm{mL}$ kanamycin (Gibco, USA), and incubated at $32^{\circ} \mathrm{C}$. After six weeks, $1 \%$ pyrocatechol (Sigma-Aldrich, USA) was dropped on colonies to select those containing the plasmid. Three different yellow colonies were picked up from the transformant, and cultivated in liquid medium containing kanamycin $25 \mu \mathrm{g} / \mathrm{mL}$ at $32^{\circ} \mathrm{C}$. Individual cultures were plated on solid medium containing $25 \mu \mathrm{g} / \mathrm{mL}$ kanamycin, $2 \%$ sucrose (Fisher Scientific, USA), and cultivated at $39^{\circ} \mathrm{C}$. After four weeks, $1 \%$ pyrocatechol was dropped on colonies to select those that might be double crossover (DCO) strains. Nine white colonies were inoculated in liquid medium containing 25 $\mu \mathrm{g} / \mathrm{mL}$ kanamycin, and cultivated at $37^{\circ} \mathrm{C}$ for three weeks. Genomic DNA was isolated and PCRs were carried out using gene-specific screening primers forward ( 5 ' - ttcaggaaacgagcgaaggt-3') and reverse (5'-gtgctatccggcggacac-3') to determine whether the WT or the KO strain was present in the targeted chromosomal region (Fig. 1B).

Construction of the M. tuberculosis complemented strain - In order to obtain the CP strain with the iunH gene, the $\mathrm{KO}$ strain was transformed by electroporation with about $2 \mu \mathrm{g}$ of the complementing plasmid construction, pNIP40::iunH. Electrocompetent cells were prepared as described above. Bacteria were plated on solid
A

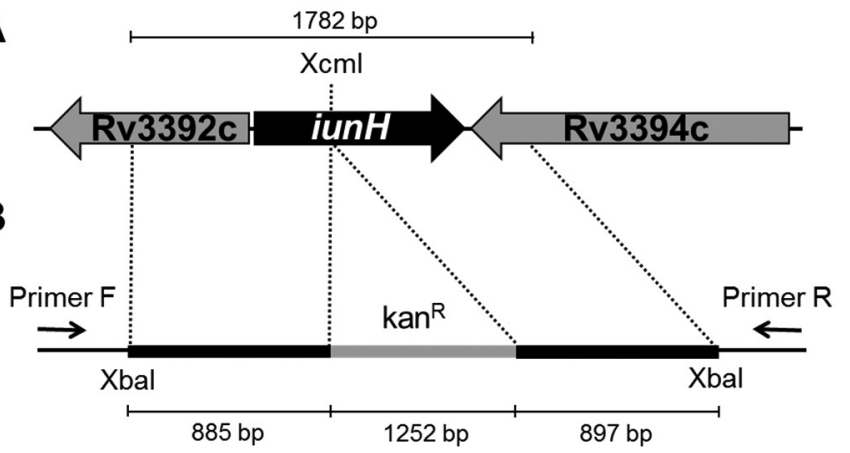

C

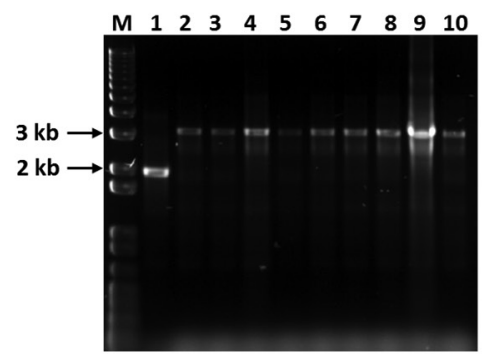

Fig. 1: genomic environment of iunH gene in Mycobacterium tuberculosis (A), regions cloned into pPR27xylE vector (B), and agarose gel electrophoresis of polymerase chain reaction (PCR) products from knockout strains (C). (A) Genomic region of iunH gene (927 bp) containing unique internal XcmI site and flanking genes; (B) the iunH gene and flanking regions (1782 bp) were amplified by PCR from $M$. tuberculosis H37Rv genomic DNA, and the iunH gene was disrupted by the insertion of a kanamycin cassette $\left(\mathrm{kan}^{\mathrm{R}}\right)$ into $X c m I$ site $\left(\right.$ iunH::kan $\left.{ }^{\mathrm{R}}\right)$. The iunH:: $\mathrm{kan}^{\mathrm{R}}$ fragment was cloned into pPR27xylE vector using XbaI restriction site. Annealing regions of gene-specific screening primers forward (Primer F) and reverse (Primer R) for the possible knockout strains of iunH gene are indicated; (C) agarose gel electrophoresis of PCR products from knockout strains which were transformed with pPR27xylE::iunH kan. M - molecular marker 1 kb plus DNA Ladder (Invitrogen), PCRs were carried out with: 1 - M. tuberculosis H37Rv genomic DNA, Lanes 2 to 10 - possible knockout strains genomic DNA. 
medium containing $50 \mu \mathrm{g} / \mathrm{mL}$ hygromycin (Invitrogen, USA) and $25 \mu \mathrm{g} / \mathrm{mL}$ kanamycin, and incubated at $37^{\circ} \mathrm{C}$. After three weeks, a single colony was cultivated in 5 $\mathrm{mL}$ of liquid medium with $50 \mu \mathrm{g} / \mathrm{mL}$ hygromycin $25 \mu \mathrm{g} /$ $\mathrm{mL}$ kanamycin at $37^{\circ} \mathrm{C}$.

Protein extraction - WT, KO and CP strains were grown in $50 \mathrm{~mL}$ liquid medium containing the proper antibiotics until an optical density at $600 \mathrm{~nm}\left(\mathrm{OD}_{600}\right)$ of 0.5-0.7. Cellular pellets were washed twice using 10 $\mathrm{mM}$ Tris $\mathrm{HCl} \mathrm{pH}$ 8.0. Cells were resuspended in $1 \mathrm{~mL}$ of the same buffer containing protease inhibitor cocktail (Promega, USA), and then transferred to $2 \mathrm{~mL}$ lysing matrix $\mathrm{B}$ tubes containing $0.1 \mathrm{~mm}$ diameter silica beads (MP Biomedicals, USA). Cells were disrupted using a L-Beader 3 (Loccus, Brazil) at a speed setting of $4000 \mathrm{rpm}, 10$ cycles of $30 \mathrm{~s}$ each, cooling between cycles. After lysis, the cell free supernatants were collected by centrifugation at $2300 \mathrm{xg}$ for $10 \mathrm{~min}$ at room temperature. The supernatants were filtered through $0.22 \mu \mathrm{M}$ Millex Durapore (Millipore, USA), and triton X-114 (Sigma-Aldrich, USA) extraction was carried out to obtain detergent and aqueous fractions as described previously (Malen et al. 2010).

Western blot - Anti-MtIAGU-NH mouse polyclonal antibody was produced by immunising a mouse with 50 $\mu \mathrm{g}$ of MtIAGU-NH purified protein (Wink et al. 2013) containing Freund's incomplete adjuvant (Sigma-Aldrich, USA) (total volume of $100 \mu \mathrm{L}$ ) by subcutaneous route, followed by a booster injection after one month. After one more month, mouse was euthanised by deep isoflurane inhalation, and blood was collected by the descendant aorta. Serum was separated by centrifugation at 10,000 $\mathrm{x} g$, for $10 \mathrm{~min}$, aliquoted, and storage at $-80^{\circ} \mathrm{C}$. Mycobacterial proteins from detergent fraction $(50 \mu \mathrm{g})$ were loaded on sodium dodecyl sulphate $12 \%$ polyacrylamide gels (SDS-PAGE), and transferred to nitrocellulose membranes (iBlot Invitrogen, USA). Blots were blocked with $5 \%$ non-fat dried milk (Santa Cruz Biotechnology, USA) $0.05 \%$ tween-20 (Sigma-Aldrich, USA) in TBS (T-TBS), and probed with anti-MtIAGU-NH polyclonal mouse antibody in a 1:500 dilution. Membranes were washed three times with T-TBS, and alkaline phosphatase-conjugated anti-mouse secondary antibody (Invitrogen, USA) was used at a dilution of 1:5000. Chemiluninescent substrate (Novex by Life Technologies, USA) was used for detection with ChemiDoc (Bio-Rad, USA).

$L C-M S / M S$ - The presence of MtIAGU-NH in the WT, KO and CP strains was also investigated by liquid chromatography coupled to mass spectrometry (LCMS/MS) of SDS-PAGE slices. Sections from 28-40 kDa of each lane/strain were excised and submitted to ingel digestion (Shevchenko et al. 2006). LC-MS/MS of peptides was performed on an Eksigent nanoLC Ultra 1D plus with AS-2 autosampler, coupled to a LTQ-XL Orbitrap Discovery (Thermo Scientific, USA). Peptide mixtures from SDS-PAGE slices were separated in reversed phase gradients in C18 (5 $\mu \mathrm{M}$ ODS-AQ C18 Yamamura Chemical Lab) column. Samples were analysed in technical triplicates. Mass spectra were acquired in a top 8 data-dependent manner with dynamic exclusion applied, and were searched against a non-redundant $M$. tuberculosis database for candidate peptides with the software Comet (Eng et al. 2012) in the platform PatternLab for Proteomics (Carvalho et al. 2016). The validity of the peptide spectra matches was assessed using the module Search Engine Processor from Patternlab for Proteomics, with a false discovery rate of $1 \%$. Identified peptides from MtIAGU-NH were manually validated and peak areas of selected peptides were calculated with Skyline (MacLean et al. 2010).

In vitro characterisation - Growth curves were determined in Sauton's medium containing $0.025 \%$ tyloxapol (Sigma-Aldrich, USA), in duplicates, at $37^{\circ} \mathrm{C}, 80 \mathrm{rpm}$ until reach the stationary phase. Aliquots were removed from each culture at different time points and the $\mathrm{OD}_{600}$ was determined. The data were evaluated with the twoway ANOVA analysis, followed by Bonferroni's post-test, using GraphPad Prism 5.0. Differences were considered significant at the $95 \%$ level of confidence. In order to quantify the intracellular concentration of the bases (uracil, guanine, adenine, and hypoxanthine) and nucleosides (uridine, guanosine, adenosine, and inosine) in the WT, $\mathrm{KO}$ and CP strains, bacteria were grown in Sauton's medium until an $\mathrm{OD}_{600}$ of $0.6-1.0$. Cells were washed twice with $1 \mathrm{x}$ PBS, and autoclaved at $121^{\circ} \mathrm{C}$ for $30 \mathrm{~min}$, followed by sonication. An HPLC method (Dionex Ultimate 3000, Thermo Scientific, USA) to identify and quantify bases and nucleosides was modified from Wink et al. (2013). Solution A ( $1 \%$ acetic acid) was maintained at $100 \%$ for $20 \mathrm{~min}$, followed by a linear gradient of $2 \mathrm{~min}$ up to $80 \%$ B (20 mM ammonium acetate) and 20\% C (methanol and acetonitrile $1: 1, \mathrm{v} / \mathrm{v}$ ) for $10 \mathrm{~min}$. The absorbance was measured at $262 \mathrm{~nm}$, injection volume was $50 \mu \mathrm{L}, 0.5 \mathrm{~mL} /$ $\mathrm{min}$, and measurements were performed in triplicates.

Macrophage infection - Macrophage infection experiments are often used to determine mycobacterial strains virulence (Copenhaver et al. 2004, Katti et al. 2008). RAW 264.7 macrophage cell line was cultured in DMEM (Gibco, USA), supplemented with 10\% heat inactivated fetal bovine serum (FBS) and $1 \%$ penicillinstreptomycin at $37^{\circ} \mathrm{C}$ with $5 \% \mathrm{CO}_{2}$. Before infection procedures, macrophages were seeded in 24-well culture plates at a density of $10^{5}$ cells per well in DMEM medium with $10 \% \mathrm{FBS}$ and incubated for $24 \mathrm{~h}$ at $37^{\circ} \mathrm{C}$ with $5 \% \mathrm{CO}_{2}$. Two independent experiments were performed, one with non-activated and the other with activated macrophages, which were activated by the addition of $5 \mathrm{ng}$ / $\mathrm{mL}$ of interferon- $\gamma$ (IFN- $\gamma$ ) (R\&D systems, USA) during $24 \mathrm{~h}$. Infection of RAW 264.7 cells with WT, KO and CP strains was performed at a multiplicity of infection of $2: 1$ (bacteria/macrophage) at $37^{\circ} \mathrm{C}$ with $5 \% \mathrm{CO}_{2}$. After $18 \mathrm{~h}$, infection was terminated by removing the overlaying medium, and each well was washed twice with sterile $0.9 \% \mathrm{NaCl}$ solution to remove extracellular bacteria. At $18 \mathrm{~h}$ after infection, and two, three, six, seven and 10 days of incubation, wells were washed with sterile $0.9 \% \mathrm{NaCl}$ solution, and the infected macrophages were then lysed with $0.025 \%$ SDS (Rodrigues Jr et al. 2014). DMEM media was changed after every three days of 
incubation. Lysates were serially diluted and plated on solid medium. Bacterial colony formation was evaluated after incubation of plates for three weeks at $37^{\circ} \mathrm{C}$. These experiments were performed in triplicates of each time point. The results were expressed as mean numbers of the logarithms of CFU per well, and were evaluated with the two-way ANOVA analysis, followed by Bonferroni's post-test, using GraphPad Prism 5.0. Differences were considered significant at the $95 \%$ level of confidence.

\section{RESULTS AND DISCUSSION}

Construction of the M. tuberculosis knockout and complemented strains - Among the nine clones screened for the $\mathrm{KO}$ of $i u n H$ gene, all suffered a double-crossover gene replacement event (Fig. 1C). To construct the CP strain, the pNIP40::iunH construction was transformed in the KO strain. The stability of the mutation introduced by gene replacement in M. tuberculosis was evaluated by plating $\mathrm{KO}$ and $\mathrm{CP}$ strains on media with and without antibiotics. The difference between the colonies obtained on plates containing antibiotics was not significant when compared with the ones obtained on plates without antibiotic, which indicates that the introduced mutation is stable (data not shown).

Evaluation of iunH knockout at protein level - As shown in Fig. 2A, a $32.9 \mathrm{kDa}$ band was detected by Western blot assay in extracts from WT and CP strains but was absent in the $\mathrm{KO}$ strain. The identity of the protein was further confirmed by LC-MS/MS from SDS-PAGE slices (28-40 kDa). Spectra matching MtIAGU-NH peptides were identified in WT and $\mathrm{CP}$ but not in KO extracts (Fig. 2B), confirming that the protein observed in WT and $\mathrm{CP}$ extracts by western blot is in fact MtIAGU-NH. As shown in Fig. 2B, the areas of the peptides identified on the WT sample are approximately 3 -fold higher than the areas identified on CP sample. No peptides were identified on the $\mathrm{KO}$ sample; consequently peak areas could not be calculated. Fig. $2 \mathrm{C}$ shows a representative MS/MS spectrum of the peptide LASVCGSSPVMR, identified in both WT and CP samples. These results indicated that the disruption of $i u n H$ gene abolishes the expression of MtIAGU-NH protein in KO strain, and the MtIAGU-NH protein expression was restored in CP strain.

In vitro characterisation - The growth rate of the $\mathrm{WT}, \mathrm{KO}$, and $\mathrm{CP}$ strains were compared to determine whether iun $H$ disruption lead to alterations during in vitro cultivation. Sauton's is a defined medium that was used for in vitro characterisation experiments to avoid interferences with traces of bases and nucleosides from $7 \mathrm{H} 9$ medium. As shown in Fig. 3A, the deletion of iunH gene led to a delay of growth in Sauton's medium during log phase. The iunH KO strain entered the early log phase of growth $\left(\mathrm{OD}_{600}\right.$ of 0.2$)$ six days later than WT strain (inset in Fig. 3A). No significant differences were found during lag and stationary phases of growth between WT and KO strains (Fig. 3A). Two independent experiments were performed, and similar results were obtained. The intracellular concentration of the bases and nucleosides was quantified in the WT, KO and CP strains. Uracil, guanine, adenine, hypoxanthine, uridine, inosine, guanosine and adenosine presented retention times of 10.53, 13.35, 14.34, 16.98, 19.89, 29.51, 29.64, and 30.36 minutes respectively. The calibration curves for all analyses were made from 0.48 to $62.5 \mu \mathrm{M}$ and all presented a correlation coefficient above 0.99 . No significant difference was observed in intracellular concentrations of either bases or nucleosides among the three strains when grown in Sauton's medium (Fig. 3B). The
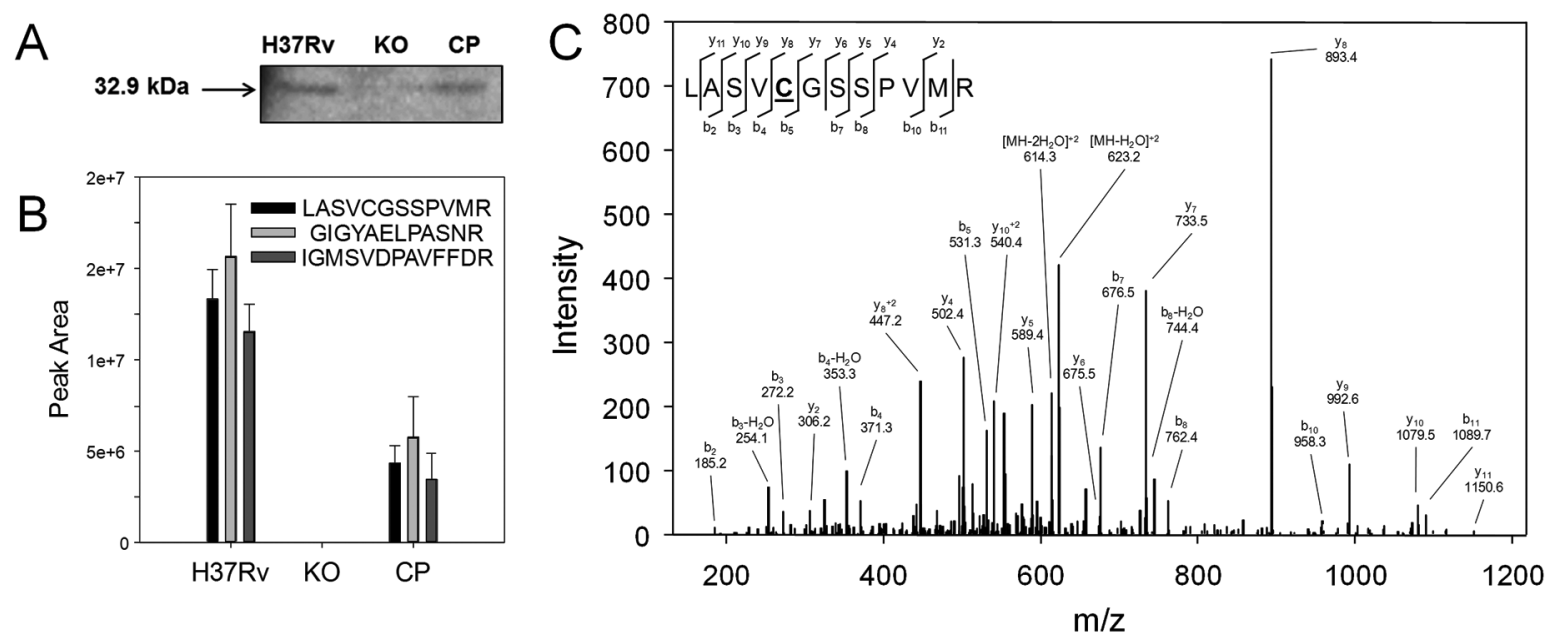

Fig. 2: evaluation of MtIAGU-NH expression. (A) Western blot analysis of protein extracts $(50 \mu \mathrm{g})$ from detergent fractions of wild-type H37Rv $(\mathrm{H} 37 \mathrm{Rv})$, knockout (KO), and complemented (CP) strains. Bands were detected by incubation with anti-MtIAGU-NH polyclonal antibody (1:500) followed by alkaline phosphatase-conjugated anti-mouse secondary antibody (1:5000); (B) peak areas of peptides LASVCGSSPVMR $\left(\mathrm{m} / z\right.$ 632.3128 $\left.{ }^{+2}\right)$, GIGYAELPASNR $\left(\mathrm{m} / z\right.$ 624.3226 $\left.6^{+2}\right)$ and IGMSVDPAVFFDR $\left(\mathrm{m} / z 543.8024^{+2}\right)$ were calculated using Skyline. LC-MS/MS analyses were performed 28-40 kDa section of SDS-PAGE samples. MS/MS spectra of identified peptides were manually validated; (C) representative MS/MS spectrum of the peptide LASVCGSSPVMR. The peptide was identified in both WT and CP samples. Doubly charged parent ion with neutral loses $\left(\left[\mathrm{MH}-\mathrm{H}_{2} \mathrm{O}\right]^{+2}\right.$ and $\left[\mathrm{MH}-2 \mathrm{H}_{2} \mathrm{O}\right]^{+2}$ ) and fragment $\mathrm{b}$ - and y-ions and their neutral loses are indicated. The cysteine (underlined) is carbamidomethylated. 
A

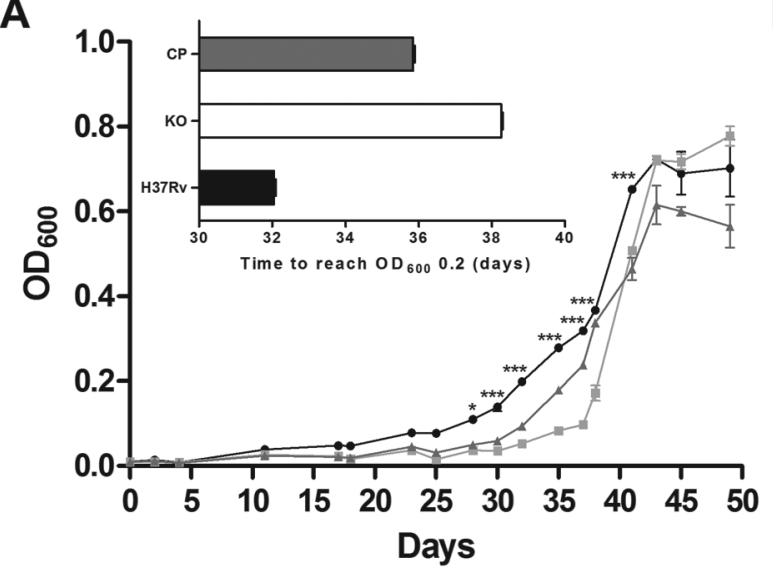

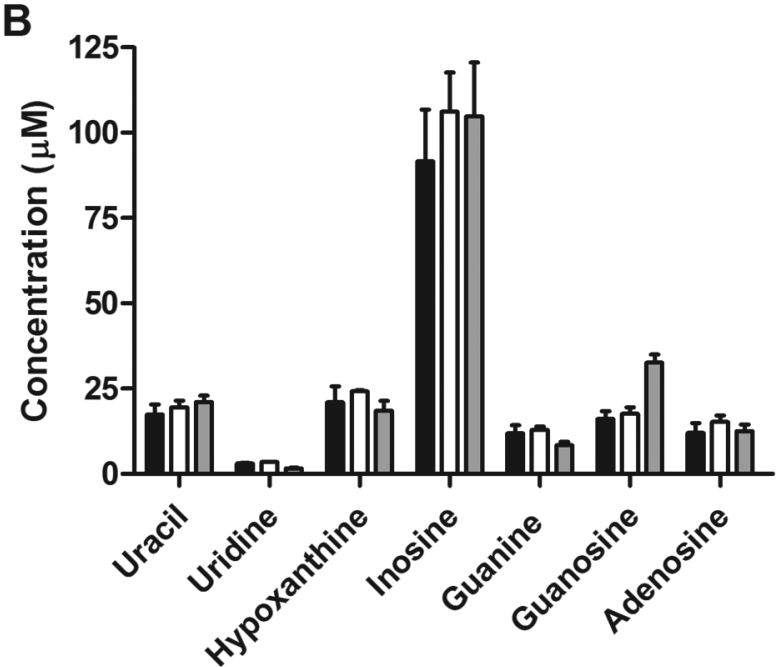

Fig. 3: in vitro characterisation of iunH knockout strain. Growth curve of WT (circle), KO for iunH gene (square), and CP (triangle) Mycobacterium tuberculosis strains grown in Sauton's medium (A); to start growth curves the different strains were diluted to reach an $\mathrm{OD}_{600}$ of 0.01 . Inset shows the time required for each strain to reach an $\mathrm{OD}_{600}$ of 0.2 . Asterisks represent significant differences between $\mathrm{WT}$ and $\mathrm{KO}$ strains by the Bonferroni post-test, ${ }^{*} p<0.05,{ }^{* *} p<0.001$. Intracellular concentrations of bases (uracil, guanine, adenine, and hypoxanthine) and nucleosides (uridine, guanosine, adenosine, and inosine) in WT (black bar), KO (white bar), and CP (grey bar) M. tuberculosis strains grown in Sauton's medium (B); adenine was not detected, and it was not included in graph; all measurements were performed in triplicates.

A

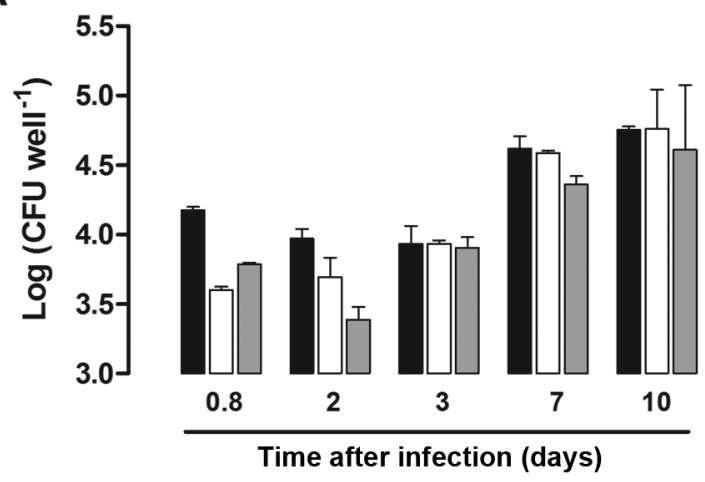

B

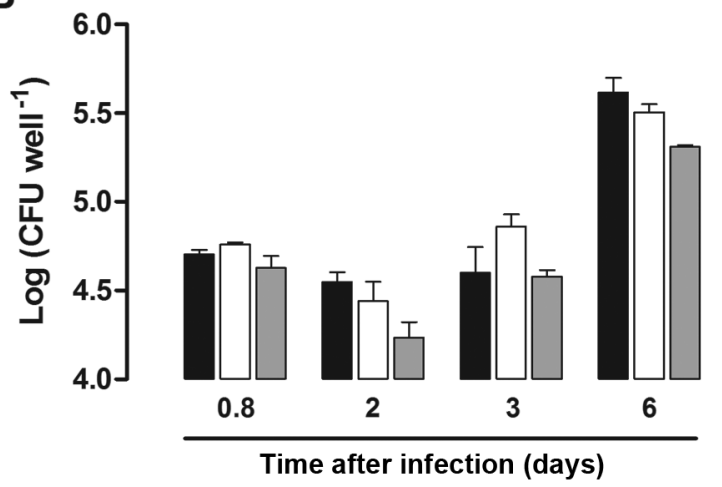

Fig. 4: infection of non-activated (A) and interferon- $\gamma$ activated (B) murine macrophages with the wild-type H37Rv (black bar), knockout (white bar) and complemented (grey bar) strains from Mycobacterium tuberculosis. The data are expressed as mean numbers of the logarithms of CFU per well of each strain of three independent measurements.

absence of accumulation of purine bases and nucleosides in iunH KO strain could be explained by the redundancy found in nucleotide metabolism of M. tuberculosis. There are at least five enzymes in nucleotide salvage pathway that use MtIAGU-NH substrates or products: purine nucleoside phosphorylase (Rv3307), hypoxanthine-guanine phosphoribosyltransferase (Rv3624c), adenine phosphoribosyltransferase (Rv2584c), uracil phosphoribosyltransferase (Rv3309c), and pyrimidine nucleoside phosphorylase (Rv3314c) (Ducati et al. 2011, Villela et al. 2011); which might compensate for the absence of $i u n H$ gene in $\mathrm{KO}$ strain.

Macrophage infection - To examine whether the iunH gene was important for invasion and growth in phagocytic cells, we determined the bacterial loads of the WT, KO and CP strains by using the macrophage model of infection. Non-activated macrophages were infected with $1.4 \times 10^{5}, 2.2 \times 10^{5}$, and $2.0 \times 10^{5} \mathrm{CFU}$, while activated cells were infected with $2.6 \times 10^{5}, 2.5 \times 10^{5}$, and $2.6 \times 10^{5} \mathrm{CFU}$ of WT, KO and CP strains, respectively, as determined at the day of infection. As shown in Fig. 4A, no significant difference was observed in intracellular growth among WT, KO, and CP strains in non-activated macrophages after $18 \mathrm{~h}$, two, three, seven and 10 days after infection. Similar results were obtained with IFN- $\gamma$ activated macrophages, where no significant difference in bacterial load of KO strain was observed when compared with both WT and CP strains after $18 \mathrm{~h}$, two, three and six days after infection (Fig. 
4B). The disruption of iun $H$ gene does not affect the $M$. tuberculosis growth in both non-activated and IFN- $\gamma$ activated RAW 264.7 cells. The concentration of $5 \mathrm{ng} / \mathrm{mL}$ of IFN- $\gamma$ or lower was shown to cause the activation of the endocytic pathway during the immune activation of RAW 264.7 (Pei et al. 2015), to induce the upregulation of the pro-inflammatory cytokine IL-18 in RAW 264.7 mouse macrophages (Kim et al. 2000), and to lead to its own production in mouse peritoneal macrophages (Di Marzio et al. 1994). In order to evaluate cytokine expression by non-activated macrophages infected with WT, KO and CP strains, the supernatants from $18 \mathrm{~h}$, and three days post infection were collected and interleukin (IL)- $1 \beta$, tumor necrosis factor (TNF)- $\alpha$, and IFN- $\gamma$ were quantified by ELISA using a commercial kit (data not shown). TNF- $\alpha$ and IL-1 $\beta$ levels of expression were not significantly different among groups, while IFN- $\gamma$ expression was not detected in culture medium of cells infected with all strains (data not shown).

In this work, we constructed a M. tuberculosis KO strain for $i u n H$ gene, validated the gene deletion at protein level, characterised the KO strain in vitro, and evaluated its ability to invade and grow in non-activated and IFN- $\gamma$ activated macrophages. The absence of accumulation of purine bases and nucleosides in M. tuberculosis iunH KO strain in Sauton's medium, together with the fact that iunH gene is not important for M. tuberculosis virulence in macrophages, could be explained by the redundancy found in nucleoside/nucleotide metabolism of M. tuberculosis. As mentioned previously, there are at least five enzymes in nucleotide salvage pathway from $M$. tuberculosis that use MtIAGU-NH substrates or products (Ducati et al. 2011, Villela et al. 2011). These enzymes might compensate for the absence of iun $\mathrm{H}$ gene in $\mathrm{KO}$ strain, consequently maintaining the nucleotide pool within the cell. Our results indicated that MtIAGU$\mathrm{NH}$ is not a target for drug development.

\section{ACKNOWLEDGEMENTS}

To Dr Mary Jackson and Dr Brigitte Gicquel, for providing the pNIP40/b and pPR27xylE plasmids.

\section{AUTHORS' CONTRIBUTION}

ADV constructed and validated the Mycobacterium tuberculosis knockout and complemented strains and wrote the manuscript; ADV and VSRJ performed macrophage infection experiments; AFMP carried out LC-MS/MS experiments; PLW and ZASQ constructed the plasmids; ADV and GOP performed in vitro characterisation; $M M C$ participated in the design of the study and statistical analysis; LAB contributed in the analysis of results and revised the manuscript; and DSS conceived the study and participated in its design and coordination.

\section{REFERENCES}

Carvalho PC, Lima DB, Leprevost FV, Santos MD, Fischer JS, Aquino PF, et al. Integrated analysis of shotgun proteomic data with PatternLab for proteomics 4.0. Nat Protoc. 2016; 11(1): 102-17.

Copenhaver RH, Sepulveda E, Armitige LY, Actor JK, Wanger A, Norris SJ, et al. A mutant of Mycobacterium tuberculosis $\mathrm{H} 37 \mathrm{Rv}$ that lacks expression of antigen $85 \mathrm{~A}$ is attenuated in mice but retains vaccinogenic potential. Infect Immun. 2004; 72(12): 7084-95.

Di Marzio P, Puddu P, Conti L, Belardelli F, Gessani S. Interferon gamma upregulates its own gene expression in mouse peritoneal macrophages. J Exp Med. 1994; 179(5): 1731-6.

Ducati RG, Breda A, Basso LA, Santos DS. Purine salvage pathway in Mycobacterium tuberculosis. Curr Med Chem. 2011; 18(9): 1258-75.

Eng JK, Jahan TA, Hoopmann MR. Comet: an open source tandem mass spectrometry sequence database search tool. Proteomics. 2012; 13(1): 22-4.

Freitas-Vieira A, Anes E, Moniz-Pereira J. The site-specific recombination locus of mycobacteriophage Ms6 determines DNA integration at the tRNA(Ala) gene of Mycobacterium spp. Microbiology. 1998; 144(12): 3397-406.

Katti MK, Dai G, Armitige LY, Marrero CR, Daniel S, Singh CR, et al. The Delta fbpA mutant derived from Mycobacterium tuberculosis H37Rv has an enhanced susceptibility to intracellular antimicrobial oxidative mechanisms, undergoes limited phagosome maturation and activates macrophages and dendritic cells. Cell Microbiol. 2008; 10(6): 1286-303.

Kim YM, Im JY, Han SH, Kang HS, Choi I. IFN-gamma up-regulates IL-18 gene expression via IFN consensus sequence-binding protein and activator protein-1 elements in macrophages. J Immunol. 2000; 165(6): 3198-205.

MacLean B, Tomazela DM, Shulman N, Chambers M, Finney GL, Frewen B, et al. Skyline: an open source document editor for creating and analyzing targeted proteomics experiments. Bioinformatics. 2010; 26(7): 966-8.

Malen H, Pathak S, Softeland T, de Souza GA, Wiker HG. Definition of novel cell envelope associated proteins in Triton X-114 extracts of Mycobacterium tuberculosis H37Rv. BMC Microbiol. 2010; 10: 132.

Parish T, Stocker NG. Electroporation of mycobacteria. In: Parish T, Stocker NG, editors. Methods in molecular biology mycobacteria protocols. Totowa: Humana Press Inc; 1998. 129-44.

Pei G, Schnettger L, Bronietzki M, Repnik U, Griffiths G, Gutierrez MG. Interferon- $\gamma$-inducible Rab20 regulates endosomal morphology and EGFR degradation in macrophages. Mol Biol Cell. 2015; 26(17): 3061-70.

Pelicic V, Jackson M, Reyrat JM, Jacobs Jr WR, Gicquel B, Guilhot C. Efficient allelic exchange and transposon mutagenesis in $M y$ cobacterium tuberculosis. Proc Natl Acad Sci USA. 1997; 94(20): 10955-60.

Rodrigues Jr V, dos Santos A, Villela AD, Belardinelli JM, Morbidoni HR, Basso LA, et al. IQG-607 abrogates the synthesis of mycolic acids and displays intracellular activity against Mycobacterium tuberculosis in infected macrophages. Int J Antimicrob Agents. 2014; 43(1): 82-5.

Shevchenko A, Tomas H, Havlis J, Olsen JV, Mann M. In-gel digestion for mass spectrometric characterization of proteins and proteomes. Nature Protocols. 2006; 1(6): 2856-60.

Villela AD, Sánchez-Quitian ZA, Ducati RG, Santos DS, Basso LA. Pyrimidine salvage pathway in Mycobacterium tuberculosis. Curr Med Chem. 2011; 18(9): 1286-98.

WHO - World Health Organization. Global tuberculosis report 2016. Geneva: WHO; 2016.

Wink PL, Sánchez-Quitian ZA, Rosado LA, Rodrigues Jr VS, Petersen GO, Lorenzini DM, et al. Biochemical characterization of recombinant nucleoside hydrolase from Mycobacterium tuberculosis H37Rv. Arch Biochem Biophys. 2013; 538(2): 80-94. 